\title{
Moving Target Indication via Three-Antenna SAR with Simplified Fractional Fourier Transform
}

\author{
Wen-Qin Wang
}

\begin{abstract}
Ground moving target indiction (GMTI) is of great important for surveillance and reconnaissance, but it is not an easy job. One technique is the along-track interferometry (ATI) synthetic aperture radar (SAR), which was initially proposed for estimating the radial velocity of ground moving targets. However, the measured differential phase may be contaminated by overlapping stationary clutter, leading to errors in velocity and position estimates. As effective clutter suppression can be achieved by multiple aperture or phase center antennas, this article presents a simplified fractional Fourier transform (SFrFT) for three-antenna-based SAR GMTI applications. This approach cancels clutter with three-antenna-based methods and forms two-channel signals through which moving targets are detected and imaged. Next, the Doppler parameters of the moving targets are estimated with the SFrFT-based estimation algorithm. In this way, both target location and target velocity are acquired. Next, the moving targets are focused with one uniform imaging algorithm. The feasibility is validated by theory analysis and simulation results.
\end{abstract}

Keywords: Fractional Fourier transform (FrFT), Simplified FrFT, Along-track interfer-ometry (ATI), Displaced phase center antenna (DPCA), Ground moving targets detection (GMTD), Synthetic aperture radar (SAR), Three-antenna SAR

\section{Introduction}

Ground moving target indication (GMTI) is of great interest for surveillance and reconnaissance [1-4], but it is not an easy job because separating the moving targets' returns from stationary clutter is a technical challenge [5]. Moving target indication is twofold [6]: one is the detection of moving targets within severe ground clutter, and the other is the estimation of their parameters such as velocity and location. As such, radar clutter has received much recognition in recent years. Several clutter suppression approaches have been proposed [7], but they often require high pulsed repeated frequency (PRF), which is not desirable to avoid excessive data rate and PRF ambiguity problem.

It is well known that the moving target with a slant range velocity will generate a differential phase shift. This phase may be detected by interferometric combination of the signals from a two-channel along-track interferometry (ATI) synthetic aperture radar (SAR) system.

Correspondence: wqwang@uestc.edu.cn

School of Communication and Information Engineering, University of Electronic Science and Technology of China, Chengdu, P. R. China
The ATI SAR was initially proposed for detecting ground moving targets [8-10], which uses two antennas to detect targets by providing essentially two identical views of the illuminated scene but at slightly different time. Several interferometry SAR (InSAR)-based moving targets detection algorithms have been proposed previously [11-14]. However, the stationary clutter unavoidably corrupts the interferometric phase of the targets depending on its signal-to-clutter environment. Consequently, the imaged moving targets will be displaced in azimuth according to its radial velocity.

There have been several studies on the clutter effects on the intended signals $[15,16]$. But there remains still many unresolved problems, e.g., how to reliably estimate the target's true interferometric phase from the clutter. Moreover, in a nonhomogeneous terrain, the degree of physical overlap of the target with a bright stationary point clutter may also influence the estimation accuracy. In order to accurately estimate the target's true velocity, clutter contamination on the signal must be minimized. Precise knowledge of the interferogram's phase and amplitude statistics is very important for distinguishing 
the moving targets from the clutter. A straightforward approach to clutter cancelation is the displaced phase center antenna (DPCA) technique [17]. For one twoantenna DPCA system, the additional freedom provided by the second antenna can be used to cancel the clutter; however, it can no longer be used to estimate the moving targets' position information.

Moreover, estimating the moving targets' Doppler parameters is often required, but the Wigner-Vill distribution-based algorithms will generate cross-terms [18], particularly in the presence of multiple moving targets. In this case, the fractional Fourier transform (FrFT) is a powerful tool. But the conventional FrFT is redundant for moving targets detection [19]. This article presents a simplified FrFT (SFrFT) and threeantenna SAR combined GMTI approach. After canceling the stationary clutter using three-antenna ATI SAR, two-channel signals through which moving targets can be detected are formed. Next, one SFrFTbased algorithm is presented to estimate the Doppler parameters of the moving targets. Finally, the moving targets are located through two-channel interferometric processing algorithm. The remaining sections are organized as follows. Section II introduces the SFrFT and its mathematical properties. Section III describes the system scheme of DPCA-based threeantenna ATI SAR for GMTI applications. Next, the SFrFT-based detection algorithm is detailed in Section IV, followed by decorrelation discussion in Section V. Finally, Section VI concludes the whole paper.

\section{Simplified Fractional Fourier Transform (FrFT)}

The FrFT is a generalization of regular Fourier transform in that the Fourier transform transforms a signal from time-domain to frequency-domain, the FrFT transforms it into a fractional Fourier domain, which is a hybridized time-frequency domain. The transform kernel of the conventional FrFT is defined as [20]

$$
K_{\alpha}(t, \mu)=\left\{\begin{array}{l}
\sqrt{\frac{1-j \cot \alpha}{2 \pi}} e^{j \frac{t^{2}+\mu^{2}}{2} \cot \alpha-j \mu t \csc \alpha}, \alpha \neq n \pi \\
\delta(t-\mu), \alpha=2 n \pi \\
\delta(t+\mu), \alpha=(2 n+1) \pi
\end{array}\right.
$$

where $n$ denotes an integer, and $\alpha$ indicates the rotation angle in FrFT domain. This operation can be considered as a generalized form of Fourier transform that corresponds to a rotation over an arbitrary angle $\alpha=$ $a \pi / 2$ with $a \in \mathfrak{R}$, as shown in Figure 1 . The forward and inverse FrFT of $x(t)$ are defined, respectively, by

$$
\chi_{\alpha}(\mu)=\int_{-\infty}^{\infty} x(t) K_{\alpha}(t, \mu) \mathrm{d} t
$$

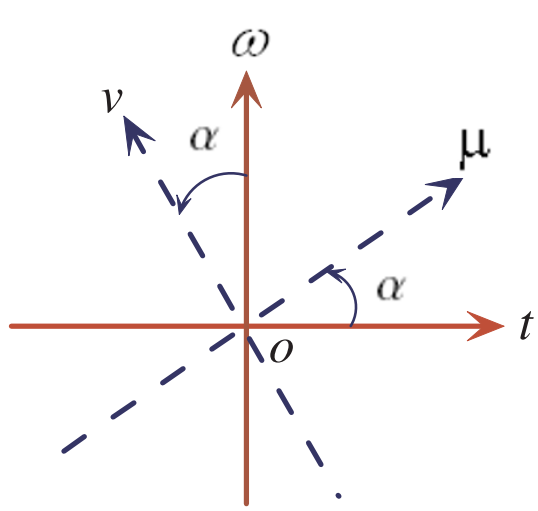

Figure 1 Time-frequency plane and a set of coordinates $(\mu, v)$ rotated by an angle $a$ relative to the original coordinates $(t, \omega)$.

$$
x(t)=\int_{-\infty}^{\infty} \chi_{\alpha}(\mu) K_{-\alpha}(t, \mu) \mathrm{d} u .
$$

Given that $\mathbf{F}$ is the Fourier transform operator and $F_{r}^{\alpha}$ is the fractional Fourier transform operator, then the FrFT possesses the following important properties.

1) Zero rotation: $F_{r}^{0}=I$ rotation: $F_{r}^{2 \pi}=I$.

2) Consistency with Fourier transform: $F_{r}^{\pi / 2}=F$.

3) Additivity of rotations: $F_{r}^{\beta} \cdot F_{r}^{\alpha}=F_{r}^{\beta+\alpha}$.

4) Linearity: $F_{r}^{\alpha}\left[c_{1} f(t)+c_{2} g(t)\right]=c_{1} F_{r}^{\alpha}(f(t))+c_{2} F_{r}^{\alpha}(g(t))$.

Additional properties can be found in the Ref. [20]. The domains $0<\alpha<\pi / 2$ are called as the fractional Fourier domains. The FrFT of a function $x(t)$, with an angle $\alpha$, can be computed as the following steps.

Step 1 . A product by a chirp:

$$
g(t)=x(t) e^{-j \frac{1}{2} t^{2} \tan \left(\frac{\alpha}{2}\right)} .
$$

Step 2. A Fourier transform (with its argument scaled by $\csc (\alpha)$ ) or a convolution:

$$
g^{*}(t)=g(t) \circledast e^{-j \frac{1}{2} t^{2} \csc (\alpha)}=\int_{-\infty}^{\infty} x(t) e^{-j \frac{1}{2}(\mu-t)^{2} \csc (\alpha)} \mathrm{d} t .
$$

where $\circledast$ denotes a convolution operator.

Step 3. Another product by a chirp:

$$
f(t)=e^{-j \frac{1}{2} \mu^{2} \tan \left(\frac{\alpha}{2}\right)} g^{*}(t) .
$$

Step 4. A product by a complex amplitude factor:

$$
F_{r}^{\alpha}[x(t)]=\sqrt{\left(\frac{1-j \cot (\alpha)}{2 \pi}\right)} f(t) .
$$


As the Steps 3 and 4 are redundant for signal detection, we name the FrFT without the Steps 3 and 4 as simplified FrFT (SFrFT). Note this SFrFT is different the simplified FrFT proposed in [21]. The SFrFT is also a linear transform and continuous in the angle $\alpha$. This provides us a powerful tool for detecting SAR moving targets, particularly when there are multiple moving targets.

\section{Three-Antenna DPCA-Based SAR Operation Scheme \\ 3.1 Background}

Consider an ATI SAR (see Figure 2) consists of two antennas moving along the azimuth direction $X$ and suppose that the two antennas are separated along the azimuth direction. For simplicity and without loss of generality, we assume that there are two interfering point targets, one moving and one stationary. Suppose they are perfectly compressed in the two SAR images, the signals reflected from the moving target and received by the two antennas can be expressed, respectively, by [15]

$$
\begin{aligned}
& s_{t 1}=A_{t 1} \delta\left(x-X_{t 1}\right) \delta\left(y-Y_{t 1}\right) \exp \left(j 4 \pi \frac{R_{t 1}}{\lambda}\right), \\
& s_{t 2}=A_{t 2} \delta\left(x-X_{t 2}\right) \delta\left(y-Y_{t 2}\right) \exp \left(j 4 \pi \frac{R_{t 2}}{\lambda}\right),
\end{aligned}
$$

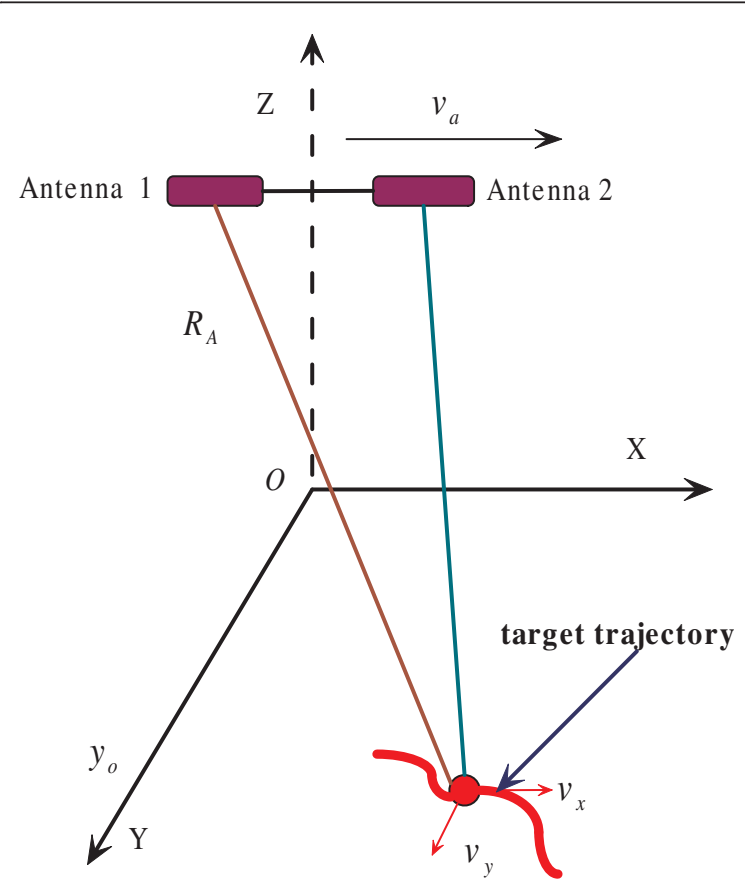

Figure 2 Along-track interferometry SAR geometry. where $A_{t i}$ is the combined gain with azimuth-compression gain and backscatter coefficient, $\left(X_{t i}, Y_{t i}\right)$ is the target position in the imaged images, $\lambda$ is wavelength, and $R_{t i}$ is the instantaneous slant range modified by convolving with the azimuth reference function. Note that in an actual system the moving point target cannot be well focused with the stationary-terrain matched filter. Similarly, the signals reflected from the stationary target and received by the two antennas are

$$
\begin{aligned}
& s_{c 1}=A_{c 1} \delta\left(x-X_{c 1}\right) \delta\left(y-Y_{c 1}\right) \exp \left(j 4 \pi \frac{R_{c 1}}{\lambda}\right), \\
& s_{c 2}=A_{c 2} \delta\left(x-X_{c 2}\right) \delta\left(y-Y_{c 2}\right) \exp \left(j 4 \pi \frac{R_{c 2}}{\lambda}\right) .
\end{aligned}
$$

Suppose the two point targets are overlaped with each other, i.e., $X_{t i}=X_{c i}$ and $Y_{t i}=Y_{c i}$, after registering the SAR image interferometric processing yields

$$
\begin{aligned}
s_{A T 1}= & \left(s_{t 1}+s_{c 1}\right) \cdot\left(s_{t 2}+s_{c 2}\right)^{*} \\
= & A_{t 1} A_{t 2} \exp \left(j 4 \pi\left(\frac{R_{t 1}-R_{t 2}}{\lambda}\right)\right)+A_{c 1} A_{c 2} \exp \left(j 4 \pi\left(\frac{R_{c 1}-R_{c 2}}{\lambda}\right)\right) \\
& +A_{t 1} A_{c 2} \exp \left(j 4 \pi\left(\frac{R_{t 1}-R_{c 2}}{\lambda}\right)\right)+A_{c 1} A_{12} \exp \left(j 4 \pi\left(\frac{R_{c 1}-R_{t 2}}{\lambda}\right)\right),
\end{aligned}
$$

where * denotes a conjugate operator. The first term is the moving target's interferogram that we are wanted. The second term is the stationary target's interferogram. Its phase should be equal to zero because a stationary scene does not change with time, i.e., $R_{c 1}=R_{c 2}$. The remaining two terms are cross-terms, which come from the clutter contamination at the SAR image formation stage. As the phase angle is $2 \pi$ periodic, the two crossterms may have different phase values; hence, the effects of cross-terms on the total along-track interferometric phase are not easily predictable.

As ATI SAR output is signal power, slowly moving targets will not attenuated along with the stationary clutter when we utilize magnitude and phase information for target extraction. In the case of low signal-toclutter ratio (SCR), the ATI SAR will lose its ability to detect slowly moving targets and to correctly estimate their velocities because the system noise (additive thermal noise and multiplicative radar phase noise) scatters the stationary clutter signal around the real axis in the complex plane. If the clutter contribution is not negligible when compared to the signal power, the estimation of the target radial velocity from the contaminated interferometric phase may lead to erroneous results. When the SCR is small, this effect will become more serious for slowly moving targets and the moving targets will be indistinguishable from the clutter. Moreover, in this case, the targets' impulse responses are not normal delta functions, particularly for the moving targets which are poorly focused because of the unmatched azimuth- 
compression filter. This leads to a point target's response overlaps with several neighboring resolution cells. This also means a varying SCR across the target's response, which in turn affects its interferometric phase. Therefore, the ATI SAR is a clutter limited moving targets detector and applying some efficient clutter suppression or cancelation techniques is necessary.

\subsection{Three-Antenna ATI SAR Scheme}

To improve the performance of slowly moving targets detection, it is necessary to apply some clutter suppression or cancelation suppression techniques. The DPCA is just proposed for this aim, which synthesizes a static antenna system allowing cancelation of static returns on a pulse-to-pulse basis. However, for the two-antenna DPCA system, after clutter cancelation it can not be used to locate the moving targets. As such, this article uses one three-antenna DPCA SAR scheme, as shown in Figure 3. An antenna of length $L$ is used as a single aperture in transmission and is split into three receiving sub-apertures in reception. In this case, the receive phase centers are displaced in the along-track direction by $L / 3$. It is then effective to define the 'two-way' phase centers as the mid-points between the phase center of the whole transmit antenna and the phase center of the receive sub-apertures. So, all goes as if the radar samples were collected by the transmit and receive antennas with phase centers co-located in the two-way phase centers. To reach aim, it is assumed that the pulse repeated frequency (PRF) is matched to the platform velocity and the distance between the three receive sub-aperture phase centers. Under this condition, the two-way phase center of the trailing sub-aperture occupies the same location of the two-way phase center of the leading subaperture one pulse repetition interval (PRI) later.

When the DPCA condition is matched, the clutter cancelation can then be performed by subtracting the samples of the radar returns received by two-way phase centers in the same spatial position, which are

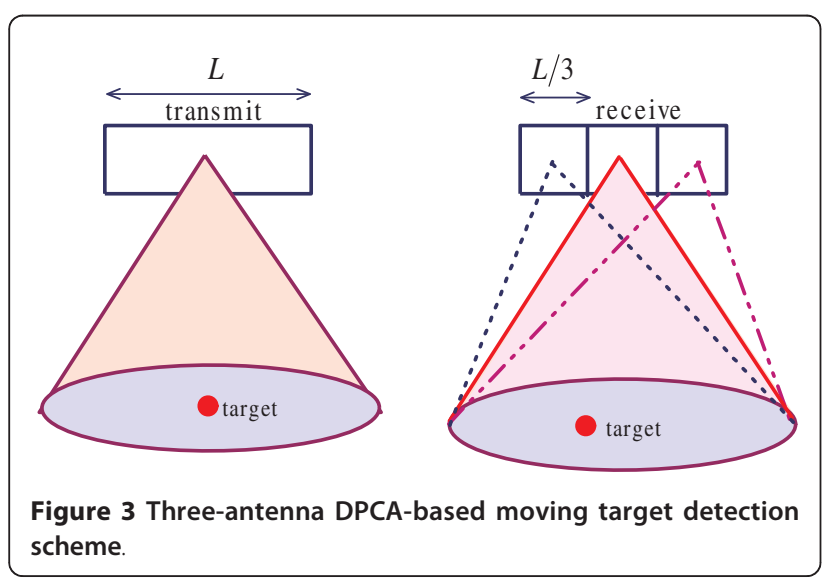

temporally displaced. The radar returns corresponding to stationary objects like the clutter from natural scenes are canceled, while the returns backscattered by moving targets have a different phase in the two acquisitions and remain uncanceled. Therefore, all static clutter scatterers are canceled, leaving only moving targets and a much simplified target detection problem (which is detailed in the next section). If the DPCA condition is not matched, the collected azimuth samples will be spaced nonuniformly. This problem can be solved using the reconstruction filtering algorithm detailed in [22].

\subsection{Signal Models}

We make the assumptions of far-field, flat earth, free space, and single polarization for our model. Although DPCA SAR can be realized with airborne and spaceborne platforms, we restrict ourselves to airborne platform only. Figure 4 shows the geometry of the threeantenna DPCA-based moving target detection (MTD) system. The platform altitude and velocity are $h$ and $v_{a}$, respectively.

The range history from the central aperture to a specific moving target located in $\left(x_{o}, y_{o}\right)$ with velocity $\left(v_{x}, v_{y}\right)$ can be represented by

$$
\begin{aligned}
R_{c}\left(t_{a}\right) & =\sqrt{\left(x_{o}+\left(v_{a}-v_{x}\right) t_{a}\right)^{2}+\left(y_{o}+v_{y} t_{a}\right)^{2}+h^{2}} \\
& \approx R_{o}+v_{y} t_{a}+\frac{\left(x_{o}+\left(v_{a}-v_{x}\right) t_{a}\right)^{2}+\left(v_{y} t_{a}\right)^{2}}{2 R_{o}}
\end{aligned}
$$

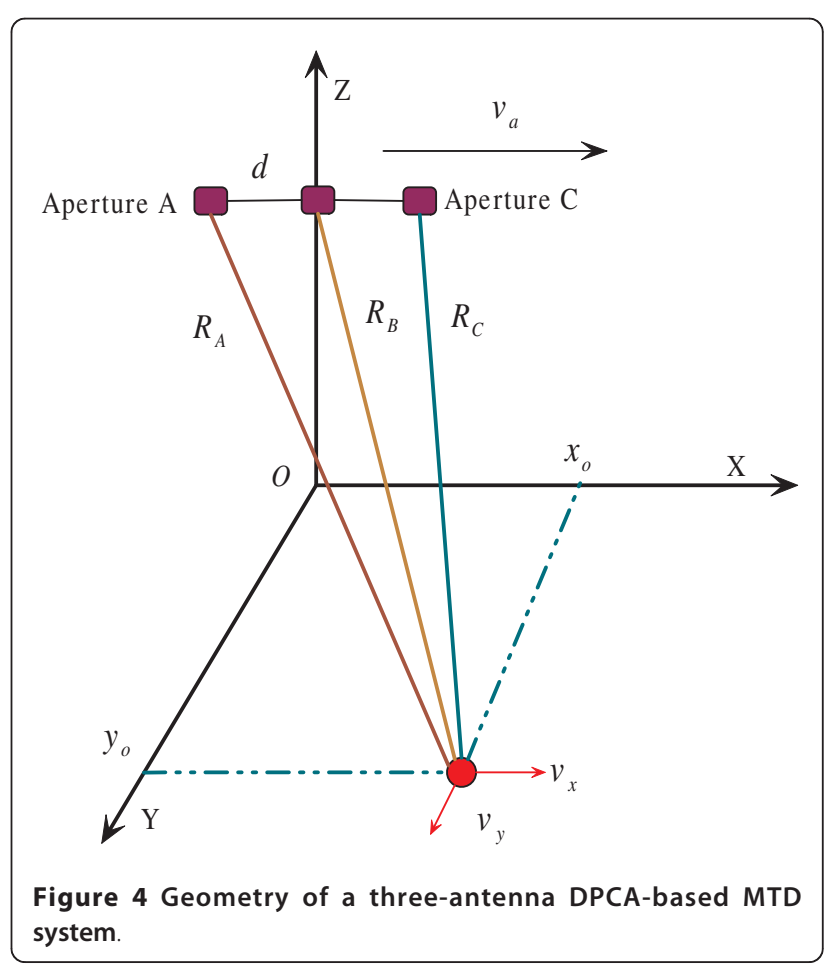


where $t_{a}$ is the azimuth time, and

$$
R_{o}=\sqrt{\gamma_{o}^{2}+h^{2}}
$$

In an alike manner, the range histories of the left aperture and the right aperture can be represented, respectively, by

$$
\begin{aligned}
& R_{l}\left(t_{a}\right) \approx R_{o}+v_{y} t_{a}+\frac{\left(x_{o}+d+\left(v_{a}-v_{x}\right) t_{a}\right)^{2}+\left(v_{y} t_{a}\right)^{2}}{2 R_{o}}, \\
& R_{r}\left(t_{a}\right) \approx R_{o}+v_{y} t_{a}+\frac{\left(x_{o}-d+\left(v_{a}-v_{x}\right) t_{a}\right)^{2}+\left(v_{y} t_{a}\right)^{2}}{2 R_{o}}
\end{aligned}
$$

with $d=L / 3$. Suppose the transmitted radar signal is

$$
s\left(t_{r}\right)=\operatorname{rect}\left[\frac{t}{T_{p}}\right] \exp \left[j 2 \pi\left(f_{c} t_{r}+\frac{1}{2} k_{r} t_{r}^{2}\right)\right],
$$

where $T_{p}$ is the pulse duration, $f_{c}$ is the carrier frequency, $k_{r}$ is the chirp rate, and $t_{r}$ is the range time. After range compressing the three-antenna SAR data, we have [23]

$$
\begin{aligned}
& S_{l}\left(t_{r}, t_{a}\right)=T_{p} \exp \left[-j 2 \pi f_{c} \xi_{l}-j \pi k_{r}\left(t_{r}-\xi_{l}\right)^{2}\right] \cdot \frac{\sin \left(k_{r} \pi\left(t_{r}-\xi_{l}\right) T_{p}\right)}{k_{r} \pi\left(t_{r}-\xi_{l}\right) T_{p}}, \\
& S_{c}\left(t_{r}, t_{a}\right)=T_{p} \exp \left[-j 2 \pi f_{c} \xi_{c}-j \pi k_{r}\left(t_{r}-\xi_{c}\right)^{2}\right] \cdot \frac{\sin \left(k_{r} \pi\left(t_{r}-\xi_{c}\right) T_{p}\right)}{k_{r} \pi\left(t_{r}-\xi_{c}\right) T_{p}}, \\
& S_{r}\left(t_{r}, t_{a}\right)=T_{p} \exp \left[-j 2 \pi f_{c} \xi_{r}-j \pi k_{r}\left(t_{r}-\xi_{r}\right)^{2}\right] \cdot \frac{\sin \left(k_{r} \pi\left(t_{r}-\xi_{r}\right) T_{p}\right)}{k_{r} \pi\left(t_{r}-\xi_{r}\right) T_{p}},
\end{aligned}
$$

where $\xi_{1}=\left(R_{l}\left(t_{a}\right)+R_{c}\left(t_{a}\right)\right) / c_{o}, \xi_{c}=2 R_{c}\left(t_{a}\right) / c_{o}$ and $\xi_{r}=$ $\left(R_{c}\left(t_{a}\right)+R_{r}\left(t_{a}\right)\right) / c_{o}$, with $c_{o}$ is the speed of light.

To cancel the clutter, we perform

$$
\begin{aligned}
& S_{c l}\left(t_{r}, t_{a}\right)=G_{1} \cdot S_{c}\left(t_{r}, t_{a}\right)-S_{l}\left(t_{r}, t_{a}+t_{d}\right), \\
& S_{r c}\left(t_{r}, t_{a}\right)=S_{r}\left(t_{r}, t_{a}\right)-G_{1} \cdot S_{c}\left(t_{r}, t_{a}+t_{d}\right),
\end{aligned}
$$

where $t_{d}=d / v_{a}$ is the relative azimuth delay between two apertures, and $G_{1}$ is used to compensate the corresponding phase shift

$$
G_{1}=\exp \left(-j \frac{2 \pi d^{2}}{4 R_{o} \lambda}\right) .
$$

where $\lambda$ is the carrier wavelength. As there is

$$
\begin{aligned}
A_{o} & =T_{p} \frac{\sin \left(k_{r} \pi\left(t_{r}-\xi_{l}\right) T_{p}\right)}{k_{r} \pi\left(t_{r}-\xi_{l}\right) T_{p}} \approx T_{p} \frac{\sin \left(k_{r} \pi\left(t_{r}-\xi_{c}\right) T_{p}\right)}{k_{r} \pi\left(t_{r}-\xi_{c}\right) T_{p}} \\
& \approx T_{p} \frac{\sin \left(k_{r} \pi\left(t_{r}-\xi_{r}\right) T_{p}\right)}{k_{r} \pi\left(t_{r}-\xi_{r}\right) T_{p}} .
\end{aligned}
$$

Equation (19a) can be expanded into [24]

$$
\begin{aligned}
S_{c l}\left(t_{r}, t_{a}\right) & =A_{o} \exp \left\{-j \frac{4 \pi}{\lambda}\left(R_{o}+v_{y} t_{a}\right)\right\} \\
& \cdot \exp \left\{\frac{x_{o}^{2}+\frac{d^{2}}{4}\left(\left(v_{a}-v_{x}\right) t_{a}\right)^{2}-2 x_{o}\left(v_{a}-v_{x}\right) t_{a}+\left(v_{y} t_{a}\right)^{2}}{2 R_{o}}\right\} \\
& \cdot\left[1-\exp \left(-j \frac{2 \pi}{\lambda} 2 v_{y} t_{d}\right)\right]
\end{aligned}
$$

From Eq. (22) we can notice that, if $v_{y}=0$, there is $S_{c l}\left(t_{r}, t_{a}\right) \mid=0$; hence, the clutter has been successfully canceled by this method. The remaining problem is to detect the moving targets.

From Eq. (22) we can derived that its Doppler frequency center and Doppler chirp rate are represented, respectively, by

$$
\begin{aligned}
& f_{d c}=-\frac{2}{\lambda}\left[v_{y}-\frac{\left(v_{a}-v_{x}\right) x_{o}}{R_{o}}\right], \\
& k_{d}=-\frac{2}{\lambda R_{o}}\left[\left(v_{a}-v_{x}\right)^{2}+v_{y}^{2}\right] .
\end{aligned}
$$

Then, equation (22) can be rewritten as

$$
S_{c l}\left(t_{r}, t_{a}\right)=A_{o}^{\prime} \exp \left[2 j \pi\left(f_{d c} t_{a}+\frac{1}{2} k_{d} t_{a}^{2}\right)+\phi_{1}\right],
$$

where

$$
\begin{aligned}
& \phi_{1}=-\frac{4 \pi}{\lambda}\left(R_{o}+\frac{x_{o}^{2}+\frac{d^{2}}{4}}{2 R_{o}}\right), \\
& A_{o}^{\prime}=A_{o}\left[1-\exp \left(-j \frac{4 \pi}{\lambda} v_{y} t_{d}\right)\right] .
\end{aligned}
$$

Thus, once the Doppler parameters described in the Eq. (25) are estimated, the target velocity $\left(v_{x}, v_{y}\right)$ can then be determined from the Eqs. (23) and (24).

To utilize the ATI technique, after compensating the phase terms caused by the relative aperture displace between $S_{c l}\left(t_{r}, t_{a}\right)$ and $S_{c r}\left(t_{r}, t_{a}\right)$ by multiplying

$$
G_{2}=\exp \left[j \frac{2 \pi}{\lambda}\left(\frac{v_{a} t_{a} d}{R_{o}}\right)\right] .
$$

From Eq. (19b) we have

$$
S_{r c}\left(t_{r}, t_{a}\right)=A_{o}^{\prime} \exp \left[2 j \pi\left(f_{d c} t_{a}+\frac{1}{2} k_{d} t_{a}^{2}\right)+\phi_{2}\right],
$$

with

$$
\phi_{2}=-\frac{4 \pi}{\lambda}\left(R_{o}+\frac{x_{o}^{2}-x_{o} d+\frac{d^{2}}{2}}{2 R_{o}}\right) .
$$


Then $S_{c l}\left(t_{r}, t_{a}\right)$ and $S_{r c}\left(t_{r}, t_{a}\right)$ can be interfered with each other by conjugate multiplication

$$
S_{c l}\left(t_{r}, t_{a}\right) S_{r c}^{*}\left(t_{r}, t_{a}\right)=\left|A_{o}^{\prime}\right|^{2} \exp \left(-\frac{2 \pi\left(x_{o} d-\frac{d^{2}}{4}\right)}{\lambda R_{o}}\right)=\left|A_{o}^{\prime}\right|^{2} \exp (\Phi),
$$

where * is the complex conjugate operator, and $\Phi$ is the interferometric phase. The azimuth position of the moving target can be determined by

$$
x_{o}=\frac{\pi d^{2}-2 R_{o} \lambda \Phi}{4 \pi d} .
$$

As the velocity of the moving target is relative small, from Eq. (13) $R_{o}$ can be approximated by

$$
R_{o}=\frac{c_{o} \hat{t}_{r}}{2}
$$

where

$$
\frac{\sin \left(k_{r} \pi\left(\hat{t}_{r}-\xi_{l}\right) T_{p}\right)}{k_{r} \pi\left(\hat{t}_{r}-\xi_{l}\right) T_{p}}=\operatorname{MAX}\left\{\frac{\sin \left(k_{r} \pi\left(t_{r}-\xi_{l}\right) T_{p}\right)}{k_{r} \pi\left(t_{r}-\xi_{l}\right) T_{p}}\right\}
$$

As there is - $W_{a} / 2 \geq x_{o} \leq W_{a} / 2, W_{a} \gg d$ with $W_{a}$ is the synthetic aperture length in azimuth, the interferometric phase is limited by

$$
\Phi|\ll \pi| \frac{ \pm W_{a} d-\frac{d^{2}}{2}}{R_{o \lambda}}|\approx| \pi \frac{W_{a}}{R_{o} \frac{\lambda}{d}} \mid \leq \pi .
$$

The interferometric phase $\Phi \in[-\pi, \pi]$ is unambiguous; hence, the unambiguous $x_{o}$ can be obtained in this way. Once $R_{o}$ and $x_{o}$ are determined, the $y_{o}$ can then be derived from the Eq. (14) because the $h$ is known from the inboard motion sensors.

\section{SFrFT-Based Moving Target Detection}

To estimate the Doppler parameters, applying the SFrFT to the Eq. (20), we get

$$
\begin{aligned}
& \chi_{\alpha}(\mu)=A_{o}^{\prime} \exp \left(j \phi_{1}\right) . \\
& \quad \int_{-T_{p} / 2}^{T_{p} / 2} \exp \left[j 2 \pi f_{d c} t_{a}+j \pi k_{d} t_{a}^{2}+j \frac{t_{a}^{2}}{2} \cot (\alpha)-j \mu t_{a} \csc (\alpha)+j \phi_{1}\right] \mathrm{d} t_{a}
\end{aligned}
$$

It arrives its maximum at [19]

$$
\begin{aligned}
& \left\{\hat{\alpha}_{0}, \hat{\mu}_{0}\right\}=\underset{\alpha, \mu}{\arg \max }\left|X_{p}(\mu)\right|^{2} \\
& \left\{\begin{array}{l}
\hat{k}_{d}=-\cot \left(\hat{\alpha}_{0}\right), \\
\hat{\alpha}_{0}=\frac{\left|X_{\hat{p}}\left(\hat{\mu}_{0}\right)\right|}{\Delta \tau} \\
\hat{f}_{d c}=\hat{\mu}_{0} \csc \left(\hat{\alpha}_{0}\right) .
\end{array}\right.
\end{aligned}
$$

This condition forms the basis for estimating the moving targets' parameters. In the SFrFT domain with a proper $\alpha$, the spectra of any strong moving target will concentrate to a narrow impulse, and that of the clutter will be spread. If we can construct a narrow band-stop filter in the SFrFT domain whose center frequency around at the center of the narrowband spectrum of a strong moving target, then the signal component of this moving target can be extracted from the initial signal. With this method, the strong moving targets can be extracted iteratively, thereafter the weak moving targets may be detectable. This method can be regarded as an extension of the CLEAN algorithm [25] to the SFrFT.

Therefore, after canceling the stationary clutter, the identification of moving targets can be implemented with SFrFT in the following steps:

Step 1. Apply one SFrFT to the data in which the clutter has been canceled with different $\alpha$, and from the maximal peak get the numerical estimation of $(\hat{\mu}, \hat{\alpha})$.

Step 2. Apply $F_{r}^{\hat{\alpha}}$ to the same data, we have

$$
X_{\hat{\alpha}}(\mu)=\chi_{\hat{\alpha}}(\mu) \text {. }
$$

Step 3. After identifying the first moving target, we then construct a narrow band-stop filter $M(\mu)$ to notch the narrow band-stop spectrum of this moving target

$$
X_{\hat{\alpha}}^{\prime}(\mu)=X_{\hat{\alpha}}(\mu) M(\mu) \text {. }
$$

Step 4. The filtered signals are then rotated back to time-domain by an inverse SFrFT.

Step 5. Repeat the operations from Step 1 to Step 4 until all the desired moving targets are identified.

Once the Doppler parameters of each target are obtained, substituting them into Eq. (23), we can get the $v_{x}$

$$
v_{y}=\frac{\left(v_{a}-v_{x}\right) x_{o}}{R_{o}}-\frac{\lambda f_{d c}}{2} \approx \frac{v_{a} x_{o}}{R_{o}}-\frac{\lambda f_{d c}}{2} .
$$

In this step, since $v_{a}, x_{o}, R_{o}, \lambda$ and $f_{d c}$ are all known variables, the $v_{y}$ can be determined successfully. In a like manner, substitute Eq. (41) into Eq. (24), we can get the $v_{x}$

$$
v_{x}=v_{a}-\sqrt{-\frac{\lambda k_{d} R_{o}}{2}-v_{\gamma}^{2}} .
$$

Note that, here $k_{d} \leq 0$ is assumed. Now, the parameters $\left(v_{x}, v_{y}\right)$ and $\left(x_{o}, y_{o}\right)$ are all determined successfully. Next, the moving targets can then be focused with one uniform image formation algorithm, such as Range-Doppler (RD) [26] and Chirp Scaling algorithms [27]. The corresponding processing steps are given in Figure 5.

\subsection{Simulation Results}

To evaluate the performance of the described processing algorithm, three-antenna DPCA stripmap SAR data from three point targets, one moving target and two stationary targets, are simulated using the parameters listed 


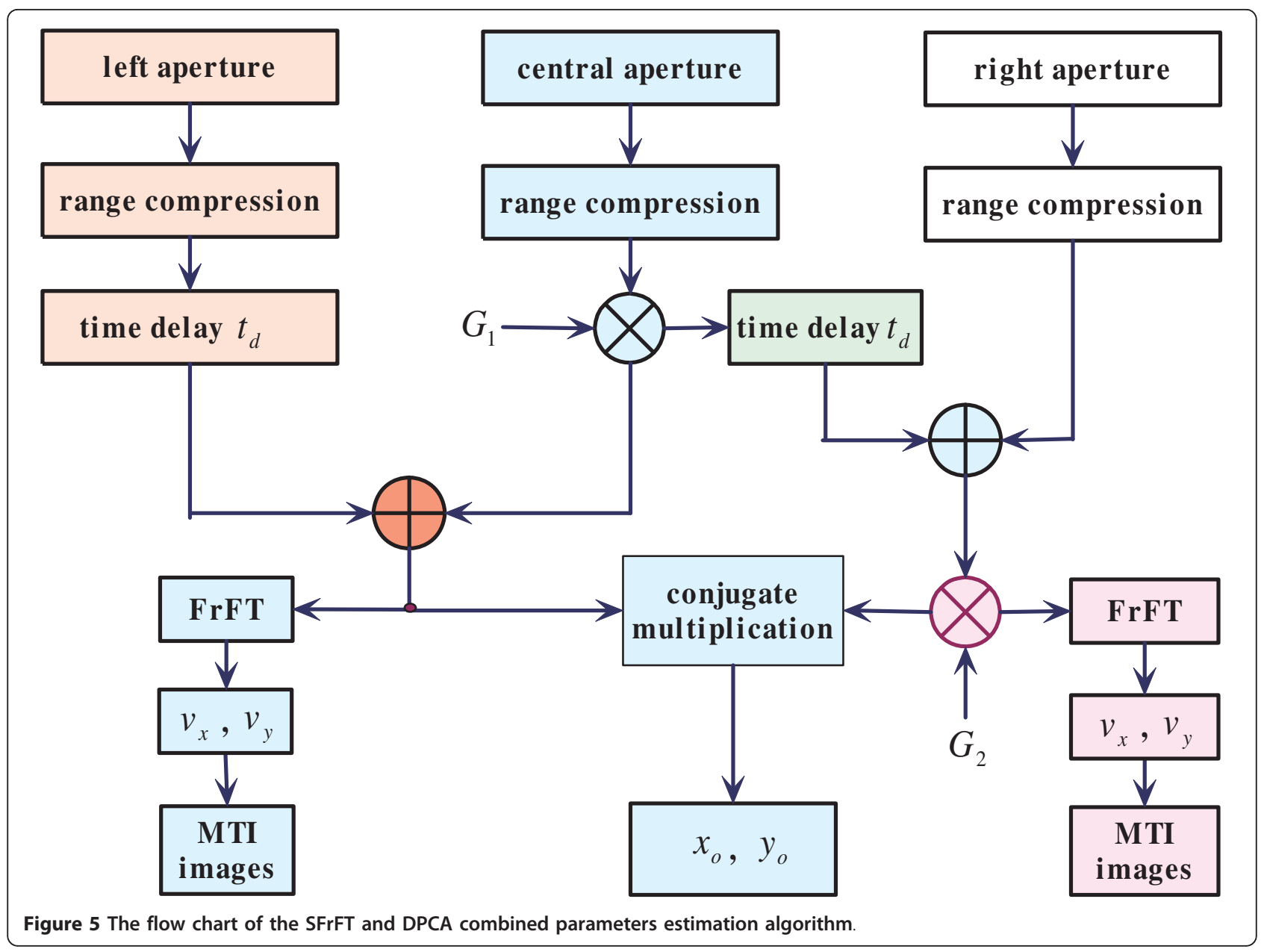

in Table 1. Figure 6 shows the processing results using the general range-Doppler imaging algorithm. It can be noticed that the imaged moving target B is overlapped with the stationary target $\mathrm{A}$. The moving target cannot be identified from this figure, because we cannot discern which is the moving one and which is the stationary one. Figure 7 shows the processing results after clutter cancelation by the DPCA operation. The clutter and

Table 1 Simulation parameters

\begin{tabular}{ccc}
\hline Parameters & Values & Units \\
\hline carrier frequency & 1.25 & $\mathrm{GHz}$ \\
pulse repeated frequency & 360 & $\mathrm{~Hz}$ \\
flying altitude & 7000 & $\mathrm{~m}$ \\
flying velocity & 180 & $\mathrm{~m} / \mathrm{s}$ \\
pulse duration & 5 & $\mathrm{fJ}, \mathrm{S}$ \\
range resolution & 5 & $\mathrm{~m}$ \\
antenna length of each aperture & 1 & $\mathrm{~m}$ \\
position of the target A & $(\mathrm{x}=50, \mathrm{y}=12000)$ & $\mathrm{m}$ \\
position of the target B & $(\mathrm{x}=58, \mathrm{y}=12000)$ & $\mathrm{m}$ \\
position of the target C & $(\mathrm{x}=50, \mathrm{y}=12250)$ & $\mathrm{m}$ \\
\hline
\end{tabular}

static returns have been canceled successfully, leaving only moving targets and a much simplified target detection problem. However, the moving target is not focused due to the improper Doppler parameters used in the range-Doppler imaging algorithm. Moreover, the imaged target position is also drifted. To focus the moving target, the accurate Doppler parameters are required.

Many algorithms considering linearly frequency modulated (LFM) signal detection, such as Wigner-Ville distribution (WVD) and Radon-Wigner transform, have been proposed. These algorithms are developed primarily for detecting single LFM signal in noise. However, they may generate cross-terms, particularly in the presence of multiple moving targets. Since cross-terms tend to oscillate more rapidly in time-frequency plane than signal auto-components, two-dimensional smoothing suppresses cross-term artifacts at the expense of decreased localization. From Figure 8, we can notice that the results contain both auto-terms and crossterms, which makes possible false detection (one more target is detected). In contrast, since FrFT is a linear transform, there will be no cross-terms. We can easily 


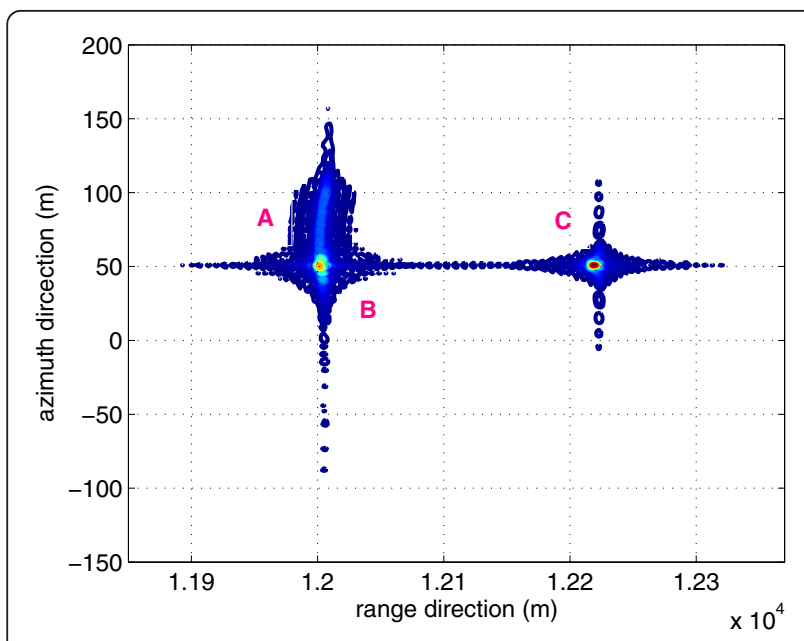

Figure 6 Processing results before clutter cancelation by DPCA operation.

locate the peak in the result of FrFT shown in Figure 9. From the peak we get ( $\mu=0.2567, \alpha=0.0092)$. Accordingly, the estimated Doppler parameters of the moving target are $\left(k_{d}=-17.34, f_{d c}=4.45\right)$. Using these estimated parameters, Figure 10 gives the corresponding imaging results. It is shown that the moving targets can be successfully focused in this way.

\section{Discussions}

In this article, the clutter cancelation is performed between two DPCA antennas. Hence, the clutter cancelation performance mainly depends on the correlation characteristics of the signals from fore and aft antennas. But phase center offset and antenna deformation may cause decorrelation. So, decorrelation analysis is necessary.

Suppose the clutter signals from the two DPCA antennas are represented by

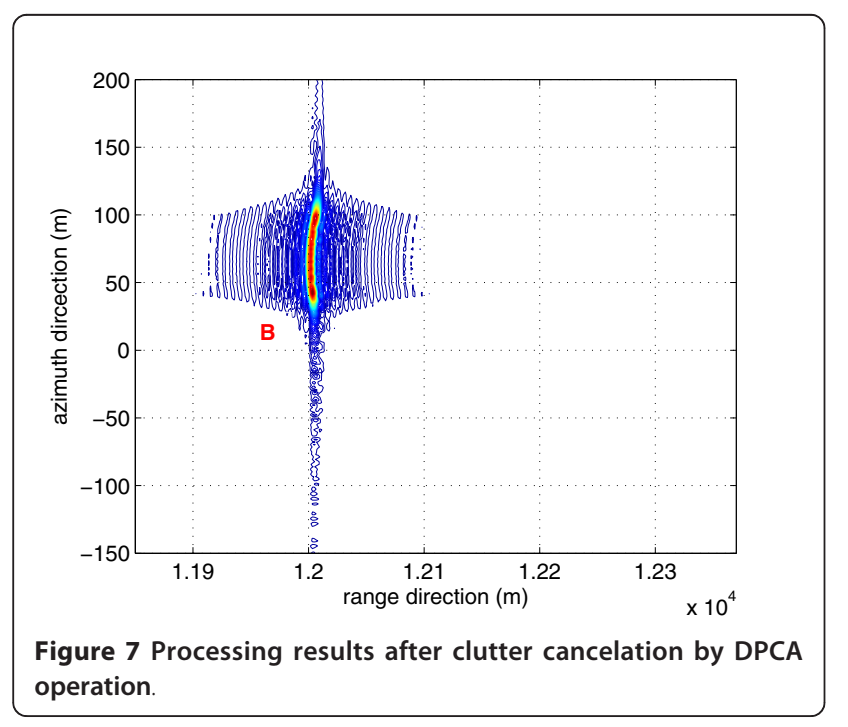

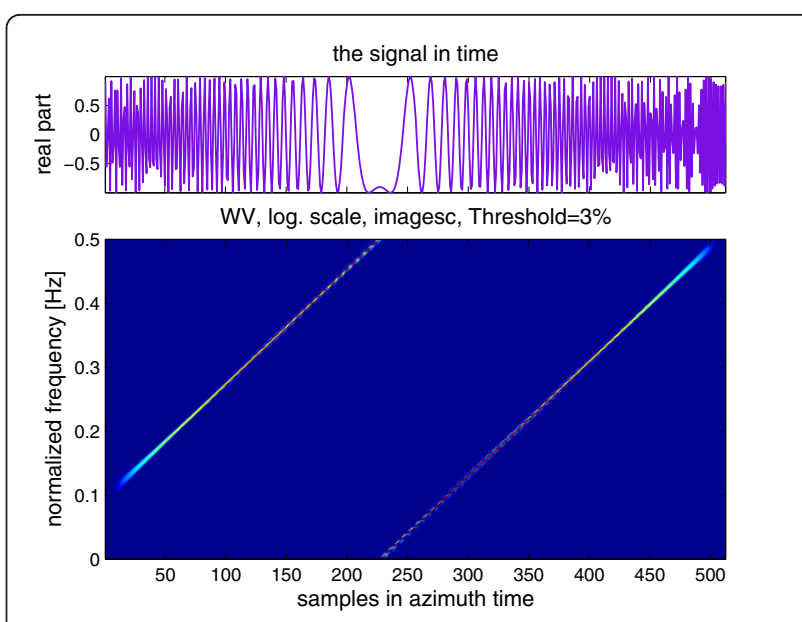

Figure 8 WVD distribution of the return of the single moving target.

$$
s_{1}=c_{1}+n_{1}, \quad s_{2}=c_{2}+n_{2},
$$

where $c_{i}$ denotes clutter signals from the $i$ th antenna, and $n_{i}$ denotes additive noise in the $i$ th antenna. The covariance matrix between the two DPCA antennas can then be represented by

$$
\begin{aligned}
R & =E\left\{\left[\begin{array}{ll}
s_{1}^{*} \cdot s_{1}, & s_{1}^{*} \cdot s_{2} \\
s_{2}^{*} \cdot s_{1}, & s_{2}^{*} \cdot s_{2}
\end{array}\right]\right\} \\
& =\left[\begin{array}{ll}
E\left\{c_{1}^{*} \cdot c_{1}+n_{1}^{*} \cdot n_{1}\right\}, & E\left\{c_{1}^{*} \cdot c_{2}\right\} \\
E\left\{c_{2}^{*} \cdot c_{1}\right\}, & E\left\{c_{2}{ }^{*} \cdot c_{2}+n_{2}^{*} \cdot n_{2}\right\}
\end{array}\right] .
\end{aligned}
$$

The multiplicative random phase noise that decorrelates the antenna 2 from the antenna 1 can be modeled as

$$
c_{2}=c_{1} \exp (j \varphi)
$$

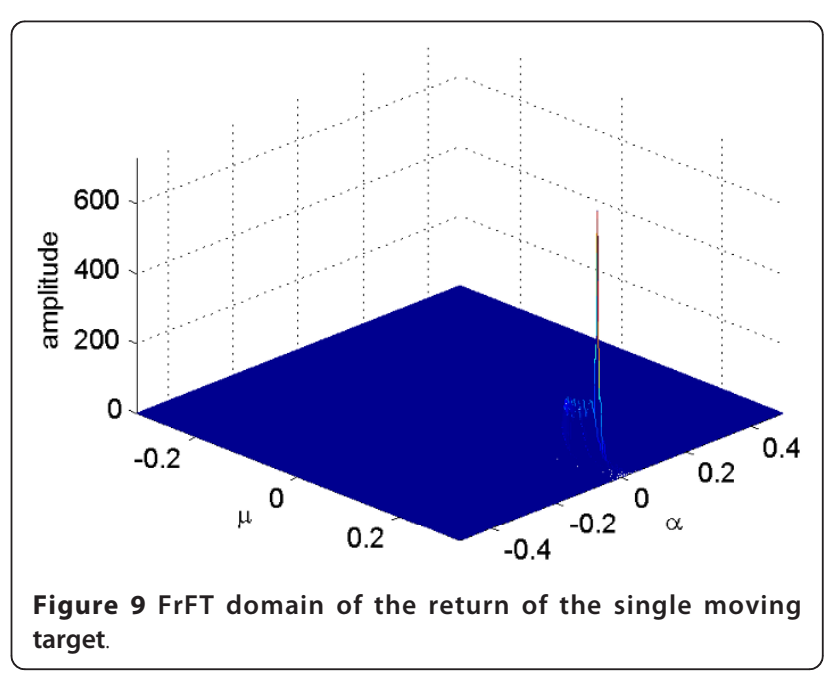




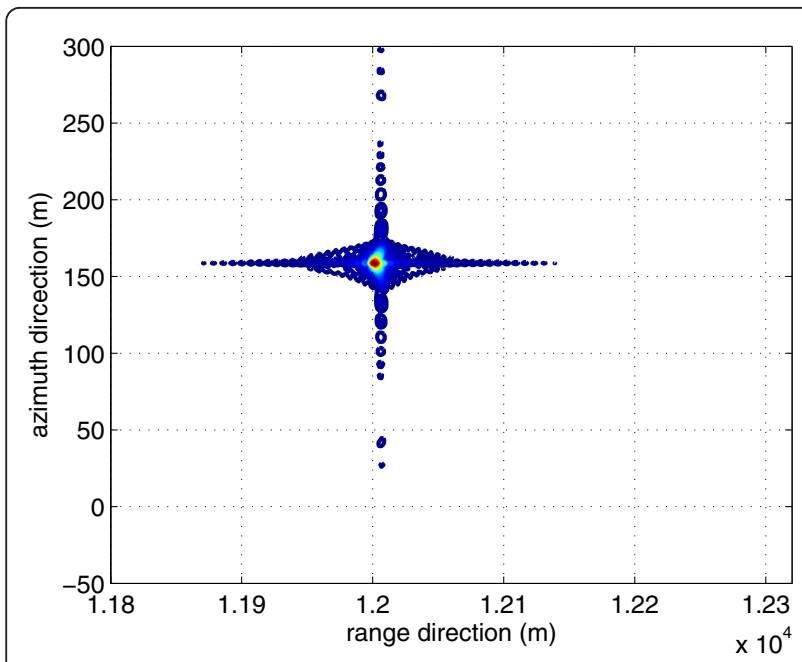

Figure 10 The focused image of the single moving target

where $c_{1}$ is deterministic and $\phi$ is normally distributed. Suppose $\phi$ with a probability density function of $f(\varphi)=N\left(0, \sigma_{\varphi}^{2}\right)$, we can get

$$
\begin{aligned}
& E\left\{c_{1}^{*} \cdot c_{1}\right\}=\xi_{c}^{2}, \quad E\left\{n_{1}^{*} \cdot n_{1}\right\}=\xi_{n}^{2}, \\
& E\left\{c_{1}^{*} \cdot c_{2}\right\}=E\left\{c_{2}{ }^{*} \cdot c_{1}\right\}=\xi_{c}^{2} \exp \left(-\sigma_{\varphi}^{2} / 2\right) .
\end{aligned}
$$

Then, Equation (44) can be further simplified as

$$
\mathbf{R}=\left[\begin{array}{lr}
\xi_{c}^{2}+\xi_{n}^{2}, & \xi_{c}^{2} \exp \left(-\sigma_{\varphi}^{2} / 2\right) \\
\xi_{c}^{2} \exp \left(-\sigma_{\varphi}^{2} / 2\right), & \xi_{c}^{2}+\xi_{n}^{2}
\end{array}\right]=\left[\begin{array}{ll}
\xi^{2}, & \xi^{2} \cdot \rho \\
\xi^{2} \cdot \rho, & \xi^{2}
\end{array}\right]
$$

where $\rho$ is the correlation coefficient, which can be determined by [28]

$$
\rho=\frac{\xi_{c}^{2} / \xi_{n}^{2}}{1+\xi_{c}^{2} / \xi_{n}^{2}} \exp \left(-\frac{\sigma_{\varphi}^{2}}{2}\right)
$$

Figure 11 shows several example correlation coefficients. We can notice that the decorrelation characteristics depend on both additive noise and multiplicative phase noise, but the DPCA clutter cancelation performance depends only on multiplicative phase noise. Thus, DPCA SAR detection performance is noise limited. In contrast, ATI SAR detection performance is clutter limited. In this article, the advantage of DPCA SAR and that of ATI SAR are combined; hence, the moving target detection performance can be improved.

\section{Conclusion}

In this article, one SFrFT and DPCA combined approach is proposed for GMTI applications. This approach realizes target location and velocity estimation with three antennas. After canceling by the three antennas, twochannel signals through which moving targets can be

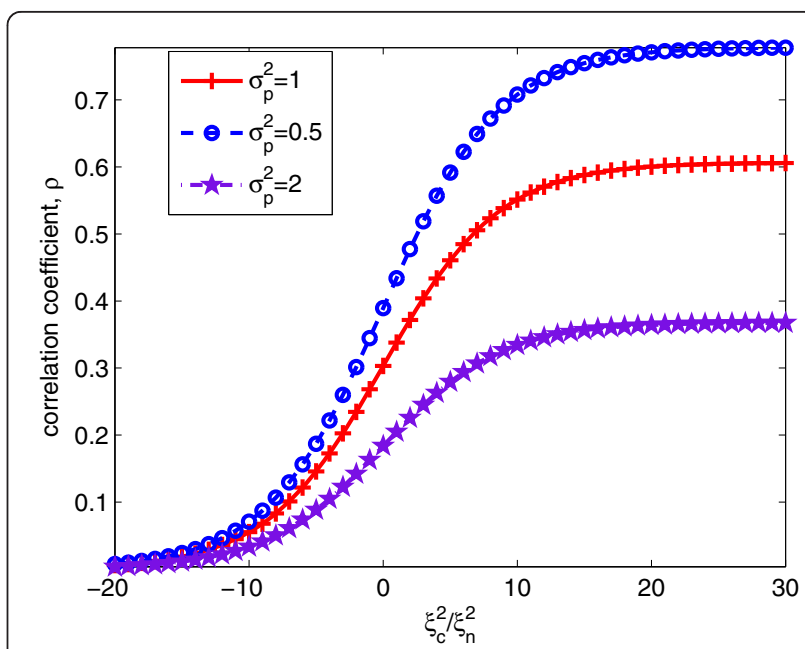

Figure 11 Example correlation coefficients as a function of clutter-to-noise ratio.

detected are formed. Next, the Doppler parameters of the moving targets are estimated with the SFrFT algorithm. Finally, the moving targets are focused with one uniform image formation algorithm. In this way, both target location and target velocity are acquired, and high-resolution moving target SAR images are obtained. Simulation results show its validity. While compared to conventional approaches, this approach is more effective and robust. In particular, it is not dependent on a target's acrosstrack velocity component or its Doppler shift, which is difficult to determine due to insufficient freedom degrees. This approach depends only on target's Doppler rate, and this is shown to be measurable with a high degree of robustness. In contrast, the conventional approaches like ATI SAR depend not only on a target's Doppler rate but also on its across-track velocity component. Moreover, the selection of matched filter length directly affects the measured ATI phase. These additional unknowns make the SAR ATI a less desirable method for estimating target parameters than the SFrFT and DPCA combined approach, which also allows the estimation of target's true azimuth position directly from its measured position in the final SAR images, particularly when there are multiple moving targets. Therefore, the SFrFT and DPCA combined method is elegant and effective in moving target identification.

\section{Acknowledgements}

This work was supported in part by the Specialized Fund for the Doctoral Program of Higher Education for New Teachers under Contract number 200806141101, and the open funds of the Key Laboratory of Ocean Circulation and Waves, Chinese Academy of Sciences under contract number KLOCAW1004.

\section{Competing interests}

The author declares that they have no competing interests. 
Received: 25 January 2011 Accepted: 24 November 2011

Published: 24 November 2011

\section{References}

1. G Li, XG Xia, YN Peng, Doppler keystone transform: an approach suitable for parallel implementation of SAR moving target imaging. IEEE Geoscience and Remote Sensing Letters 5(4), 573-577 (2008)

2. D Wu, DY Zhu, ZD Zhu, Knowledge-aided multichannel adaptive SAR/GMTI processing: algorithm and experimental results. EURASIP Journal on Advances in Signal Processing, Article ID 164187, 12 (2010)

3. JS Bergin, PM Techau, Multiresolution signal processing techniques for ground moving target detection using airborne radar. EURASIP Journal on Advances in Signal Processing, Article ID 47534, 16 (2006)

4. D Page, G Owirka, Knowledge-aided STAP processing for ground moving target indication radar using multilook data. EURASIP Journal on Advances in Signal Processing, Article ID 74838, 16 (2006)

5. WQ Wang, An approach for multiple moving targets detection and velocity estimation, in Proceedings of IEEE Radar Conference, New York, 749-753 (2006)

6. JHG Ender, CH Gierull, DC Maori, Improved space-based moving target indication via alternate transmission and receiver switching. IEEE Transactions on Geoscience and Remote Sensing 46(12), 3960-3974 (2008)

7. XM Li, DZ Feng, HW Liu, MD Xing, D Luo, Two-dimensional pulse-to-pulse canceler of ground clutter in airborne radar. IET Radar, Sonar and Navigation 3(2), 133-143 (2009). doi:10.1049/iet-rsn:20080108

8. A Budillon, V Pascazio, G Schirinzi, Estimation of radial velocity of moving targets by along-track interferometric SAR systems. IEEE Geoscience and Remote Sensing Letters 5(3), 349-353 (2008)

9. Z Z Dong, B Cai, DN Liang, Detection of ground moving targets for twochannel spaceborne SAR-ATI. EURASIP Journal on Advances in Signal Processing, Article ID 230785, 9 (2010)

10. L Yang, T Wang, $Z$ Bao, Ground moving target indication using an In-SAR system with a hybrid baseline. IEEE Geoscience and Remote Sensing Letters 5(3), 373-377 (2008)

11. AM Guarbieri, S Tebaldini, On the exploitation of target statistics for SAR interferometry applications. IEEE Transactions on Geoscience and Remote Sensing 46(11), 3436-3443 (2008)

12. R Romeiser, H Runge, S Suchandt, J Sprenger, H Weilbeer, A Sohrmann, D Stammer, Current measurements in rivers by spaceborne along-track InSAR. IEEE Transactions on Geoscience and Remote Sensing 45(12), 4019-4031 (2007)

13. R Romeiser, H Runge, Theoretical evaluation of several possible along-track InSAR modes of TerraSAR-X for ocean current measurements. IEEE Transactions on Geoscience and Remote Sensing 45(1), 21-35 (2007)

14. S Chiu, MV Dragosevic, Moving target indication via RADARSAT-2 multichannel synthetic aperture. EURASIP Journal on Advances in Signal Processing, Article ID 740130, 19 (2010)

15. S Chiu, Clutter effects on ground moving target velocity estimation with SAR along-track interferometry, in Proceedings of International IEEE Geoscience and Remote Sensing Symposium, Toulouse, France, 1314-1319 (2003)

16. A Durak, CH Gierull, Clutter effects on the interferometric phase of ground moving targets. DRDC Ottawa Research Report TM 2005-175 Defense R\&D Canada-Ottawa. (2005)

17. P Lombardo, F Colone, D Pastina, Monitoring and surveillance potentialities obtained by splitting the antenna of the COSMO-SkyMed SAR into multiple sub-apertures. IEE Proceedings on Radar, Sonar and Navigation 153(2), 104-116 (2006). doi:10.1049/ip-rsn:20045122

18. PL Shui, HY Shang, YB Zhao, Instantaneous frequency estimation based on directionally smoothed pseudo-Wigner-Vill distribution bank. IET Radar, Sonar and Navigation 1(4), 317-325 (2007). doi:10.1049/iet-rsn:20060123

19. WQ Wang, Approach of multiple moving targets detection for microwave surveillance sensors. International Journal of Information Acquisition 4(1), 57-68 (2007). doi:10.1142/S0219878907001162

20. LB Almeida, The fractional Fourier transform and time-frequency representations. IEEE Transactions on Signal Processing 42(10), 3084-3091 (1992)

21. SC Pei, JJ Ding, Simplified fractional Fourier transform. J Opt Soc Amer. 17(13), 2355-2367 (2000)

22. N Gebert, G Krieger, A Moreira, SAR signal reconstruction from nonuni-form displaced phase centre sampling in the presence of perturbations, in
Proceedings of IEEE Geoscience and Remote Sensing Symposium, Seoul, Kroea, 1034-1037 (2005)

23. WQ Wang, CB Ding, XD Liang, Time and phase synchronization via directpath signal for bistatic synthetic aperture radar systems. IET Radar Sonar and Navigation 2(1), 1-11 (2008). doi:10.1049/iet-rsn:20060097

24. MJ Zheng, RL Yang, JC Zhou, A new method of moving targets detection and imaging based on multi-phase center antenna. Modern Radar In Chinese 25(12), 55-57 (2003)

25. J Tsao, BD Steinber, Reduction of sidelobe and speckle artifacts in microwave imaging. IEEE Transactions on Antennas and Propagation 36(4), 543-556 (1988). doi:10.1109/8.1144

26. YL Neo, FH Wong, IG Cumming, Processing of azimuth-invariant bistatic SAR data using the range Doppler algorithm. IEEE Transactions on Geoscience and Remote Sensing 46(1), 14-21 (2008)

27. FH Wong, TS Yeo, New applications of nonlinear chirp scaling in SAR data processing. IEEE Transactions on Geoscience and Remote Sensing 39(5) 946-953 (2001). doi:10.1109/36.921412

28. S Chiu, C Livingstone, A comparison of displaced phase centre antenna and along-track interferometry techniques for RADARSAT-2 ground moving indication. Cana J Remote Sens. 33(1), 27-51 (2005)

doi:10.1186/1687-6180-2011-117

Cite this article as: Wang: Moving Target Indication via Three-Antenna

SAR with Simplified Fractional Fourier Transform. EURASIP Journal on

Advances in Signal Processing 2011 2011:117.

\section{Submit your manuscript to a SpringerOpen ${ }^{\mathcal{O}}$ journal and benefit from:}

- Convenient online submission

- Rigorous peer review

- Immediate publication on acceptance

- Open access: articles freely available online

- High visibility within the field

- Retaining the copyright to your article

Submit your next manuscript at $>$ springeropen.com 